\title{
Vestibular evoked myogenic potentials: optimal stimulation and clinical application $^{\dagger}$
}

\author{
Yi-Ho Young ${ }^{1,2, *}$ \\ ${ }^{1}$ Department of Otolaryngology, National Taiwan University Hospital, 1 Chang-Te Street, Taipei, Taiwan; \\ ${ }^{2}$ National Taiwan University College of Medicine, Taipei, Taiwan
}

Received 22 November 2005; accepted 14 July 2006

(c) 2006 National Science Council, Taipei

Key words: sacculo-collic reflex, sternocleidomastoid muscle, vestibular evoked myogenic potential

\begin{abstract}
Summary
By easily stimulating the ear with loud sound and recording on tonically contracted neck muscles, vestibular evoked myogenic potential (VEMP) test can reflect inner ear function other than the cochlea and semicircular canal. This expands the test battery for clinicians to explore saccular disease, adding a potential usefulness to the sacculo-collic reflex. The ideal stimulation mode for VEMPs is as follows: $95 \mathrm{~dB}$ tone bursts, frequency $500 \mathrm{~Hz}$, stimulation repetition rate $5 \mathrm{~Hz}$, rise/fall time $1 \mathrm{~ms}$, plateau $2 \mathrm{~ms}$, binaural stimulation with bilateral recordings. Animal model using guinea pigs has been established, which sets the stage for useful future studies investigating VEMPs in guinea pigs that would appear to resemble human VEMP responses. Clinically, VEMP test has been widely used in central and peripheral vestibular disorders.
\end{abstract}

\section{Introduction}

There are four nerve bundles passing through the internal auditory canal. Previously, electroneurography (ENoG), auditory brainstem response (ABR), and caloric test were utilized to evaluate the function of facial, cochlear, and superior vestibular nerves, respectively. Only the function of the inferior vestibualr nerve remains unexplored. Bickford et al. [1] demonstrated that loud sound stimuli could cause myogenic "inion response" indicative of the activation of the vestibular organs. However, it was until the revision of the recording setting by Colebatch and Halmagyi [2] that vestibular evoked myogenic potential (VEMP) became a practical clinical test. It can easily be recorded from the tonically contracting

*To whom correspondence should be addressed. Fax: + 886-223946674; E-mail: youngyh@ha.mc.ntu.edu.tw

${ }^{\dagger}$ Grant no. NSC 94-2314-B002-239 from National Science Council, Taipei, Taiwan. Presented at the 8th Japan-Taiwan Joint Conference in Otolaryngology, Taipei, December 17, 2005. sternocleidomastoid (SCM) muscle by stimulation with loud clicks or tone bursts. Colebatch et al. [3] labeled the serial peaks (p13, n23, n34, p44) of the evoked response according to their latencies, and found that wave p13-n23 was present in all normal subjects, whereas wave $\mathrm{n} 34-\mathrm{p} 44$ was only present in $60 \%$ of the subjects.

VEMPs are proposed to be generated via a disynaptic pathway, beginning in the saccular macula, then via the inferior vestibular nerve, lateral vestibular nucleus, medial vestibulospinal tract, and finally terminating on the motor neurons of the SCM muscle [4-6]. Through the efforts during past decade, VEMPs have been validated to reflect the sacculo-collic reflex, and widely used clinically.

\section{Modes of stimulation and recording}

\section{Acoustic stimulation}

The sternocleidomastoid (SCM) muscle was chosen as the target to record the VEMPs [7]. Surface 
electromyographic (EMG) activity was recorded on the subject being in a supine position. To prevent unnecessary high levels of SCM activation, a pillow was put under the head to have the neck at about $30^{\circ}$ elevated compared to the horizon. The $0.9-\mathrm{cm} \mathrm{Ag} / \mathrm{AgCl}$ electrode was attached on the upper half of the SCM muscle on both sides, and a reference electrode on the lateral end of the upper sternum. During recording, the investigator kept an eye on the monitor and the subject was instructed to keep the head elevated in the pitch plane, leading to active SCM muscle contraction at a relatively constant level $(50-200 \mu \mathrm{V})$. Acoustic stimuli (95 dBHL, short tone bursts; $500 \mathrm{~Hz}$, ramp $=1 \mathrm{~ms}$, plateau $=2 \mathrm{~ms}$ ) were given to the ear through insertion-type earphone. EMG signals were amplified and bandpass filtered between 30 and $3000 \mathrm{~Hz}$. The stimulation rate was $5 \mathrm{~Hz}$, analysis time for each response was $60 \mathrm{~ms}$, and 200 responses were averaged for each run. Two consecutive runs were performed to verify the reproducibility, and the results were averaged providing the final response $[8,9]$.

\section{Interpretation}

The initial positive/negative polarity of the waveform with peaks were termed $\mathrm{p} 13$ and $\mathrm{n} 23$ on the basis of the respective latencies (Figure 1). The latencies of peaks p13 and n23, and peak-to-peak amplitude p13-n23 were measured for evaluation. Accordingly, delayed VEMP indicates the mean latency of p13 exceeding the level (mean $+2 \mathrm{SD})$, suggesting lesion in the retrolabyrinth, especially in the vestibulo-spinal tract [10].

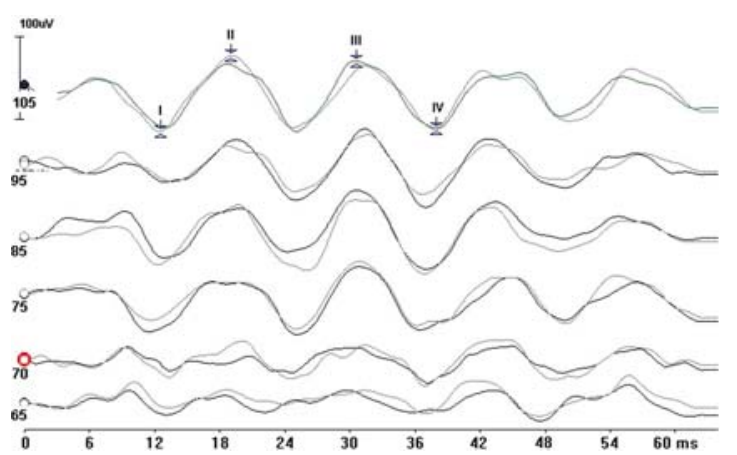

Figure 1. Myogenic potentials evoked by tone burst stimulation with different stimulus intensities in a 22-year-old man. It reveals the thresholds as $75 \mathrm{dBHL}$ for both p13-n23 and n34p44 components. (I: p13, II: n23, III: n34, IV: p44; 105: 105 dBHL, 95: 95 dBHL, 85: 85 dBHL, 75: 75 dBHL).
There is a consensus that amplitude of the VEMP depends on the stimulus intensity and voluntary muscular effort [11]. Under similar acoustic stimulation, the effectiveness of a neuromuscular reflex, either excitatory or inhibitory, can therefore be correlated with the voluntary task undertaken by the muscle [12]. Because the amplitude of the VEMP varies substantially between subjects and in one subject between trials, either interaural amplitude difference (IAD) ratio or relative amplitude was used to adjust the side difference of amplitudes [13]. The IAD ratio is defined as interaural peak-to-peak amplitude difference over the sum of amplitudes of both ears [14]. For example, if the norm of IAD ratio was $0.13 \pm 0.10$, thus IAD ratio greater than 0.33 (mean $+2 \mathrm{SD}$ ) was defined as abnormal. Relative amplitude means the amplitude of VEMPs using method A divided by that using method B.

Each subject underwent serial VEMP tests. Initial stimulus intensity started at $95 \mathrm{dBHL}$, followed by a $10-\mathrm{dB}$ step decrement, until the waveforms zwere absent. Subsequently, the stimulus intensity was increased in 5-dB steps until the VEMP response reappeared. Threshold was the level at which VEMP response was reestablished. If $95 \mathrm{dBHL}$ acoustic stimuli failed to elicit VEMP responses, then the subject underwent $105 \mathrm{dBHL}$ (machine upper limits) acoustic stimulation for further confirmation of absent VEMPs [15].

\section{Monaural vs. binaural stimulation}

In order to safe time and energy in eliciting higher response rate for VEMPs binaural tone bursts stimulation with bilateral recordings was suggested [16]. The result shows that VEMPs by binaural stimulation provide neither different information nor less variability, as compared with VEMPs by monaural stimulation. Furthermore, recording from binaural stimulation can be used as a possibly more convenient mode compared with two monaural recordings, especially when testing young or old or disabled patients, since a continuous muscular effort is required during recording [17]. Furthermore, binaural simultaneous acoustic stimulation with bilateral recording can be utilized to compare the right-left VEMP difference, and the side difference of p13-n23 amplitude is adjusted using a relative amplitude or IAD ratio [15]. 
Monaural acoustic stimulation with ipsilateral recording may elicit larger amplitude of the earlier components (p13, n23), whereas binaural acoustic stimulation with bilateral recording evokes higher response rate and larger amplitude of the later components (n34, p44). Although the nerve pathways for both components are different, the thresholds for these potentials do not differ significantly, indicating that both components may, at least in part, share a common origin, but different pathways [15].

\section{Head elevation vs. head rotation methods}

The VEMP response has the property of scaling in size in proportion to the level of tonic muscle activity. A feature, of particular importance for a reflex, is its effectiveness in matching the voluntary task being undertaken [18]. Because active and tonic SCM muscle contraction is essential for recording the VEMP responses, false-negative VEMPs are sometimes encountered in those who cannot sustain SCM muscle contraction by head elevation. Hence, effortless head rotation method to activate SCM muscle was raised to replace conventional head elevation method in the aged, newborn, or debilitated subjects.

In a supine position without a pillow, the subject rotated his/her head sideways toward one shoulder as head down on the yaw plane to activate the SCM muscle. The tone burst stimulation was given to the ear up, and VEMPs were recorded ipsilaterally. To ensure the SCM muscle activation, the subject was instructed to allow his/ her chin to touch the shoulder throughout the entire test. The EMG activities were monitored during recording to maintain muscle activities at a relatively constant level (50-200 $\mu \mathrm{V})$. Hence, applying increased effort in the head elevation method may result in a neuromuscular reflex with increased effectiveness [13], leading to large amplitude of VEMPs. However, the lower response rate with smaller amplitude prevents the use of the head rotation method as an initial screening test for VEMPs. Therefore, when VEMP responses cannot be elicited by the head elevation method, the head rotation method should be utilized to reduce false negative results [19].
Tapping evocation or bone-conduction (BC) stimulation

Halmagyi et al. [20] reported that VEMP responses are typically absent in cases of conductive hearing loss with air-bone gap measuring $\geq 20 \mathrm{~dB}$, hence the response rate of VEMPs by tone-burst method is low. In contrast, the tapping method displays higher response rate of VEMPs in patients with conductive hearing loss, indicating that the VEMP provoked by tapping with a tendon hammer on the forehead is a vibratory input rather than an auditory input [21]. The typical biphasic wave due to tapping implies that the receptive and the motor units are intact in the patients with conductive hearing loss. However, the limitation for the tapping method is non-uniform, thus has been replaced by BC method [22].

In $\mathrm{BC}$ method, tone bursts $(70 \mathrm{~dB} \mathrm{nHL}$, $500 \mathrm{~Hz}$, ramp $=1 \mathrm{~ms}$, plateau $=2 \mathrm{~ms}$ ) stimulation was given via a bone vibrator, which was placed on the tip of mastoid ipsilaterally, and recorded unilaterally. The stimulation rate was $5 \mathrm{~Hz}$ and total 200 responses were averaged for each run. Although the precise origin of boneconducted VEMPs has not been well established, it is proposed to be from the saccule [23].

\section{Galvanic stimulation}

To determine the site of lesion responsible for absent VEMPs, recently, it has been shown that galvanic stimulation also evokes myogenic responses on the SCM muscle [24]. Watson and Colebatch [25] suggested that galvanic stimulation would stimulate the most distal portion of the vestibular nerve while the clicks would act at the receptor level. The cathode electrode was on the mastoid, and the anodal electrode was on the forehead. The average responses in the unrectified EMG activities of the SCM can be elicited by galvanic stimulation ( $3 \mathrm{~mA}, 1 \mathrm{~ms})$, and be useful in the differential diagnosis of labyrinthine from retrolabyrinthine lesions in patients with absent VEMPs evoked by tones.

\section{VEMPs in experimental animals}

Although VEMP has been validated to reflect the sacculo-collic reflex, eliciting VEMP from an alert 
animal remains unexplored. Sakakura et al. [26] applied artificial respiration in decerebrated animals to ensure the muscular contraction. However, those methods are not under normal physiological condition. Alternatively, Yang and Young [27] devised a special clamp for forceful contraction of the neck muscles in guinea pigs under alert condition.

Recording of evoked potentials was performed on the neck extensors of a guinea pig at the level of third cervical vertebral bone (C3). A pair of needle electrodes was placed on neck extensors, while a reference electrode was placed on the occipital area at the midline, and EMG activity was recorded. During recording, the animal was fixed with its head elevated and neck hyperextended while in a prone position throughout the entire test. EMG signals were amplified and bandpass filtered between 30 and $3000 \mathrm{~Hz}$. Click stimuli $(100 \mathrm{dBSPL}$, duration $=0.1 \mathrm{~ms})$ were generated by an earphone connected via a short tube inserted into bilateral ear canals. Each animal was evoked by monaural acoustic stimulation with unilateral recording. The stimulation rate was $5 \mathrm{~Hz}$, analysis time for each response was $24 \mathrm{~ms}$, and 200
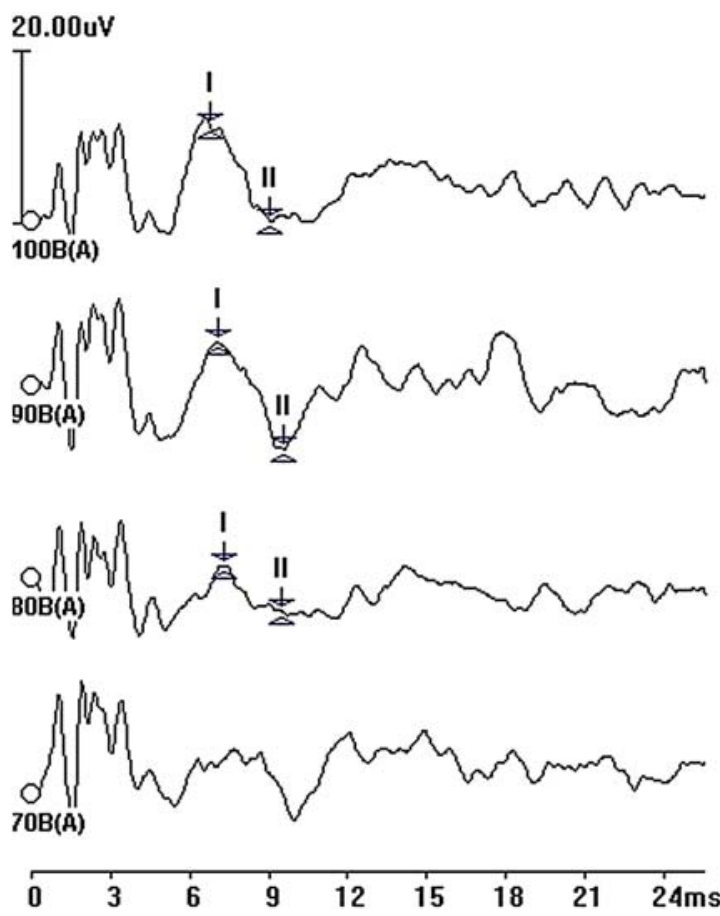

Figure 2. Click-evoked myogenic potentials in a guinea pig reveal the thresholds as $80 \mathrm{dBSPL}$. (I: positive peak; II: negative peak). responses were averaged for each run. Two consecutive runs were performed to verify its reproducibility, and the results were averaged to provide the final response. Thereafter, the latencies of positive peak, negative peak, and peak-to-peak amplitude were measured (Figure 2).

In the guinea pigs treated with gentamicin, the thresholds of ABR were not elevated. In contrast, absent caloric responses on the lesion ears accompanied by absent click-evoked myogenic potentials indicates that the vestibulo-ocular reflex and sacculo-collic reflex are affected by gentamicin, respectively. Therefore, the use of gentamicin-treated animals, along with normal controls and ABR data, resulted in convincing results that the recorded myogenic potentials were in fact of vestibular origin. It sets the stage for useful future studies investigating VEMPs in guinea pigs that would appear to resemble human VEMP responses.

\section{Clinical application}

\section{Peripheral inner ear disorders}

\section{(a) Sudden deafness}

For differentiating the origin of VEMP from cochlear or vestibular organs, $\mathrm{Wu}$ and Young [28] conducted VEMP test in 20 patients of unilateral sudden deafness without vertigo. All patients displayed normal biphasic VEMP responses, with the mean latencies of p13 and n23 non-significantly different to either the contralateral healthy ears or the normal control ears. Restated, the transduction pathway of the VEMPs is unrelated to the hearing level, i.e., the cochlear nerve.

\section{(b) Vestibular neuritis}

Chen et al. [29] studied VEMPs in eight patients with vestibular neuritis to localize the lesion site. Seven $(88 \%)$ of the eight patients had bilateral normal VEMPs, revealing sparing of the inferior vestibular nerve. Thus, an intact inferior vestibular nerve seems necessary to generate benign paroxysmal positional vertigo in the future [30]. In contrast, Murofushi et al. [31] reported that eight patients with vestibular neuritis had absence of both click and galvanic VEMPs, indicating that vestibular neuritis may affect inferior division of the vestibular nerve as well. 
(c) Herpes zoster oticus ( $H Z O$ )

Herpes zoster oticus is manifested as auricular eruption, facial palsy, and vertigo with spontaneous nystagmus beating toward the healthy side. Unlike vestibular neuritis, absent caloric and VEMPs responses may be shown [32]. Therefore, the nerve trunks within the internal auditory canal are widely affected in $\mathrm{HZO}$ patients with vertigo. Both superior and inferior divisions of the vestibular nerve attribute to the vertiginous attack.

(d) Meniere's disease

Staging of Meniere's disease proposed by AAOHNS (1995) was based on the arithmetic mean of the pure tone thresholds at $0.5,1,2$, and $3 \mathrm{kHz}$, using the worst audiogram during the interval 6 months before treatment. Since the saccule, next to the cochlea, is the second most frequent site for hydrops formation [33], VEMP response is supposed to reflect the stage of Meniere's disease [34].

Comparing the IAD ratio and the stage of Meniere's disease demonstrates a significant relationship, whereas no significant relationship exists between the $\%$ unilateral weakness of caloric response and the stage of disease. Therefore, the IAD ratio of VEMPs correlates with the stage of Meniere's disease, and can be served as another aid to assess the stage of Meniere's disease [35].

(e) Acute low-tone hearing loss ( $A L H L$ )

Acute low-tone hearing loss is termed as idiopathic low-tone sensorineural hearing loss of acute onset, with relative preserved high-tone hearing. By way of electrocochleography, the pathophysiology of ALHL is attributed to the endolymphatic hydrops. Furthermore, lack of vertigo and nystagmus in those with ALHL suggests that the endolymphatic hydrops is confined to the cochlea. Because most patients with ALHL reveal normal VEMPs throughout the episode, while half of Meniere's disease with low-tone hearing loss demonstrate abnormal VEMPs, hence, VEMP test can be used to differentiate ALHL from stage I of Meniere's disease [36].

\section{(f) Meniere attack}

Acute vertiginous attack in Meniere's disease, the so-called Meniere attack, always brings the patient to the emergent room, where spontaneous nystagmus accompanied by nausea and vomiting is observed. Two mechanisms are proposed for the
Meniere attack [37, 38]. One is membrane rupture theory, which mainly occurs on the Reissner's membrane, and the other is membrane distension theory, indicating that the utricular or saccular membrane herniates into the semicircular canal. Most patients with Meniere attacks revealed abnormal VEMPs, indicating that the saccule "participates" in the event of Meniere attack, an important idea that stimulates consideration of the mechanism of Meniere attack [34, 39]. Abnormal VEMPs may or may not normalize after Meniere attack depending on the individual saccular pathology [40, 41].

\section{(g) Superior canal dehiscence syndrome}

In addition to chronic otitis media and otosclerosis, patients with superior canal dehiscence syndrome can also have apparent conductive hearing loss, but intact VEMP responses to tones. Pathologically lowered VEMP thresholds of 55$70 \mathrm{dBHL}$, with larger amplitude on the affected side is the main characteristic of VEMP [42]. This is due to a mobile window created by the superior canal dehiscence may have enhanced the transmission of sound pressure through the vestibule that is required for activation of the sacculus [43].

\section{Central vestibular lesions}

\section{(h) Basilar type migraine}

Basilar type migraine is a distinctive form of complicated migraine characterized by neurological signs referable to the brainstem, cerebellum, or occipital cortex. It is characterized by recurrent headache, usually localized to the occipital area, associated with multiple neurological symptoms such as headache, nausea, vomiting, vertigo, gait ataxia, paresthesia, dysarthria, and dysphargia, etc [44]. Patients with basilar type migraine may display absent or delayed VEMPs, presumably because the descending pathway from the saccule, through the brainstem to the XIth cranial nerve is interrupted [45].

\section{(i) Multiple sclerosis}

Shimizu et al. [46] investigated VEMPs in patients with multiple sclerosis and reported that the latencies of a vestibulospinal reflex can be prolonged in multiple sclerosis. Prolonged VEMPs could be due to demyelination from either of 
primary afferent axons at the root entry zone or secondary vestibulospinal tract axons rather than to lesions involving vestibular nucleus [47-49]. Measurement of VEMPs is therefore helpful in detecting subclinical vestibulospinal lesions in suspected multiple sclerosis [50].

\section{(j) Spinocerebellar degeneration}

Takegoshi and Murofushi [51] recorded VEMPs in patients with spinocerebellar degeneration, including olivo-ponto-cerebellar ataxia (OPCA), cortical cerebellar atrophy (CCA), and Machado-Joseph disease (MJD). The results revealed VEMPs in patients with OPCA and CCA types of spinocerebellar degeneration were preserved well. In contrast, VEMPs were definitely abnormal in patients with MJD. It has been reported that the peripheral nervous system is frequently affected in patients with MJD. It seems more likely that the losses of the vestibulo-collic and vestibulo-ocular reflex in MJD patients might be due to the degeneration of the peripheral vestibular system.

\section{(k) Brainstem stroke}

As caloric test assesses the vestibulo-ocular reflex (VOR), which passes ascend through the upper brainstem; VEMP test evaluates the sacculo-collic reflex, which travels descend via the lower brainstem. Some patients with brainstem stroke who have normal VOR can have abnormal VEMPs, presumably because interruption of the descending pathway from the brainstem to the 11th cranial nerve which relates to sacullo-collic reflex [52]. Nevertheless, age-related effects may account for a proportion of the abnormalities, as most patients tested were over age 60 [53]. Therefore, to reduce false negative VEMP results, the head rotation method should be performed in the elderly if conventional head elevation method fails to elicit VEMP responses [19].

\section{(l) Cerebellopontine angle tumor}

The role of VEMP test in cerebellopontine angle (CPA) tumor was to evaluate its origin, either superior or inferior division of the vestibular nerve. Prior to operation, VEMP test can be employed to predict the nerve of origin, and to formulate the best surgical approach. After operation, VEMP test can be used to disclose the residual function of the inferior vestibular nerve [54-56].

\section{References}

1. Bickford R.G., Jacobson J.L. and Cody D.T.R., Nature of averaged evoked potentials to sound and other stimuli in man. Ann. N.Y. Acad. Sci. 112: 204-223, 1964.

2. Colebatch J.G. and Halmagyi G.M., Vestibular evoked potentials in human neck muscles before and after unilateral vestibular deafferentation. Neurology 42: 1635-1636, 1992.

3. Colebatch J.G., Halmagyi G.M. and Skuse F., Myogenic potentials generated by a click-evoked vestibulocollic reflex. J. Neurol. Neurosurg. Psychiatry 57: 190-197, 1994.

4. Murofushi T., Curthoys I.S., Topple A.N., Colebatch J.G. and Halmagyi G.M., Responses of guinea pig primary vestibular neurons to clicks. Exp. Brain Res. 103: 174-178, 1995.

5. Murofushi T., Curthoys I.S. and Gilchrist D.P., Response of guinea pig vestibular nucleus neurons to clicks. Exp. Brain Res. 111: 149-152, 1996.

6. Uchino Y., Sato H., Sasaki M., Ikegami H., Isu M. and Graf W., Sacculocollic reflex arcs in cats. J. Neurophysiol. 77: 3003-3012, 1997.

7. Wu C.H., Young Y.-H. and Murofushi T., Tone burstevoked myogenic potentials in human neck flexor and extensor. Acta Otolaryngol. 119: 741-744, 1999.

8. Cheng P.W., Huang T.W. and Young Y.-H., The influence of clicks versus short tone bursts on the vestibular evoked myogenic potentials. Ear Hear. 24: 195-197, 2003.

9. Murofushi T., Matsuzaki M. and Wu C.H., Short tone burst-evoked myogenic potentials on sternocleidomastoid muscle. Are these potentials also of vestibular origin?. Arch. Otolaryngol. Head Neck Surg. 125: 660-664, 1999.

10. Murofushi T., Shimizu K., Takegoshi H. and Cheng P.W., Diagnostic value of prolonged latencies in the vestibular evoked myogenic potential. Arch. Otolaryngol. Head Neck Surg. 127: 1069-1072, 2001.

11. Lim C.L., Clouston P., Sheean G. and Viannikas C., The influence of voluntary EMG activity and click intensity on the vestibular click evoked myogenic potential. Muscle Nerve 18: 1210-1213, 1995.

12. Matthews P.B.C., Observations on the automatic compensation of reflex gain on varying the pre-existing level of motor discharge in man. J. Physiol. 374: 73-90, 1986.

13. Young Y.-H. and Kuo S.W., Side-difference of vestibular evoked myogenic potentials in healthy subjects. Hear. Res. 198: 93-98, 2004.

14. Young Y.-H., Wu C.-C. and Wu C.H., Augmentation of vestibular evoked myogenic potentials - an indication for distended saccular hydrops. Laryngoscope 112: 519-522, 2002.

15. Wang C.T. and Young Y.-H., Earlier and later components of tone burst evoked myogenic potentials. Hear. Res. 191: 59-66, 2004.

16. Brantberg K. and Fransson R.-A., Symmetry measures of vestibular evoked myogenic potentials using objective detection criteria. Scand. Audiol. 30: 189-196, 2001.

17. Wang S.J. and Young Y.-H., Vestibular evoked myogenic potentials using simultaneous binaural acoustic stimulation. Hear. Res. 185: 43-48, 2003.

18. Marsden C.D., Rothwell J.C. and Day B.L., Long-latency automatic responses to muscle stretch in man: origin and function. Adv. Neurol. 39: 509-539, 1983. 
19. Wang C.T. and Young Y.-H., Comparison of the head elevation and rotation methods in eliciting vestibular evoked myogenic potentials. Ear Hear. 27: 376-381, 2006.

20. Halmagyi G.M., Yavor R.A. and Colebatch J.G., Tapping the head activates the vestibular system: a new use for the clinical reflex hammer. Neurology 45: 1927-1929, 1995.

21. Yang T.L. and Young Y.-H., Radiation-induced otitis media-study by a new test, vestibular evoked myogenic potential. Int. J. Radiat. Oncol. Biol. Phys. 60: 295-301, 2004.

22. Sheykholeslami K., Murofushi T., Kermany M.H. and Kaga K., Bone-conducted evoked myogenic potentials from the sternocleidomastoid muscle. Acta Otolaryngol. 120: 731-734, 2000.

23. Welgampola M.S., Rosengren S.M., Halmagyi G.M. and Colebatch J.G., Vestibular activation by bone conducted sound. J. Neurol. Neurosurg. Psychiatry 74: 771-778, 2003.

24. Murofushi T., Takegoshi H., Ohki M. and Ozeki H., Galvanic-evoked myogenic responses in patients with an absence of click-evoked vestibulo-collic reflexes. Clin. Neurophysiol. 113: 305-309, 2002.

25. Watson S.R.D. and Colebatch J.G., Vestibulocollic reflexes evoked by short-duration galvanic stimulation in man. J. Physiol. 513: 587-597, 1998.

26. Sakakura K., Miyashita M., Chikamatsu K., Takahashi K. and Furuya N., Tone burst-evoked myogenic potentials in rat neck extensor and flexor muscles. Hear. Res. 185: 57-64, 2003.

27. Yang T.H. and Young Y.-H., Click-evoked myogenic potentials recorded on alert guinea pigs. Hear. Res. 205: 277-283, 2005.

28. Wu C.C. and Young Y.-H., Vestibular evoked myogenic potentials are intact after sudden deafness. Ear Hear. 23: 235-238, 2002.

29. Chen C.W., Young Y.-H. and Wu C.H., Vestibular neuritis: three-dimensional videonystagmography and vestibular evoked myogenic potential results. Acta Otolaryngol. 120: 845-848, 2000.

30. Welgampola M.S. and Colebatch J.G., Characteristics and clinical applications of vestibular evoked myogenic potentials. Neurology 64: 1682-1688, 2005.

31. Murofushi T., Monobe H., Ochiai A. and Ozeki H., The site of lesion in "vestibular neuritis". Study by galvanic VEMP. Neurology 61: 417-418, 2003.

32. Lu Y.C. and Young Y.-H., Vertigo from herpes zoster oticus-superior or inferior vestibular nerve origin? Laryngoscope 113: 307-311, 2003.

33. Okuno T. and Sando I., Localization, frequency and severity of endolymphatic hydrops and the pathology of the labyrinthine membrane in Meniere's disease. Ann. Otol. Rhinol. Laryngol. 96: 438-445, 1987.

34. de Waele C., Tran Ba Huy P., Diard J.P., Fraysse G. and Vidal P.P., Saccular dysfunction in Meniere's disease. Am. J. Otol. 20: 223-232, 1999.

35. Young Y.-H., Huang T.W. and Cheng P.W., Assessing the stage of Meniere's disease using vestibular evoked myogenic potentials. Arch. Otolaryngol. Head Neck Surg. 129: 815-818, 2003.

36. Wu C.L. and Young Y.-H., Vestibular evoked myogenic potentials in acute low-tone sensorineural hearing loss. Laryngoscope 114: 2172-2175, 2004.

37. Schuknecht H.F., Pathophysiology of endolymphatic hydrops. Arch. Otorhinolaryngol. 212: 253-262, 1976.
38. Tonndorf J., Mechanical causes of fluctuating hearing loss. Otolaryngol. Clin. North Am. 8: 303-313, 1975.

39. Dohlmann G.F., On the mechanism of the Meniere attack. Arch. Otorhinolaryngol. 212: 301-307, 1977.

40. Kuo S.W., Yang T.H. and Young Y.-H., Change of vestibular evoked myogenic potentials after Meniere attack Ann. Otol. Rhinol. Laryngol. 114: 717-721, 2005.

41. Young Y.-H., Huang T.W. and Cheng P.W., Vestibular evoked myogenic potentials in delayed endolymphatic hydrops. Laryngoscope 112: 1623-1626, 2002.

42. Brantberg K., Bergenius J. and Tribukait A., Vestibularevoked myogenic potentials in patients with dehiscence of the superior semicircular canal. Acta Otolaryngol. 119: 633-640, 1999.

43. Minor L.B., Clinical manifestations of superior semicircular canal dehiscence. Laryngoscope 115: 1717-1727, 2005.

44. Baloh R.W., Neurotology of migraine. Headache 37: 615$621,1997$.

45. Liao L.J. and Young Y.-H., Vestibular evoked myogenic potentials in basilar artery migraine. Laryngoscope 114: 1305-1309, 2004.

46. Shimizu K., Murofushi T., Sakurai M. and Halmagyi G.M., Vestibular evoked myogenic potentials in multiple sclerosis. J. Neurol. Nuerosurg. Psychiatry 69: 276-277, 2000.

47. Sartucci F. and Logi F., Vestibular evoked myogenic potentials: a method to assess vestibulo-spinal conduction in multiple sclerosis patients. Brain Res. Bull. 59: 59-63, 2002.

48. Versino M., Colnaghi S., Callieco R., Bergamaschi R., Romani A. and Cosi V., Vestibular evoked myogenic potentials in multiple sclerosis patients. Clin. Neurophysiol. 113: 1464-1469, 2002.

49. Bandini F., Beronio A., Ghiglione E., Solaro C., Parodi RC. and Mazzella L., The diagnostic value of vestibular evoked myogenic potentials in multiple sclerosis. J. Neurol. 251: 617-621, 2004.

50. Tu C.E. and Young Y.-H., Audiovestibular evolution in a patient with multiple sclerosis. Ann. Otol. Rhinol. Laryngol. 113: 726-729, 2004.

51. Takegoshi H. and Murofushi T., Vestibular evoked myogenic potentials in patients with spinocerebellar degeneration. Acta Otolaryngol. 120: 821-824, 2000.

52. Chen C.H. and Young Y.-H., Vestibular evoked myogenic potentials in brainstem stroke. Laryngoscope 113: 990-993, 2003.

53. Welgampola MS. and Colebatch JG., Vestibulocollic reflexes: normal values and the effect of age. Clin. Neurophysiol. 112: 1971-1979, 2001.

54. Murofushi T., Matsuzaki M. and Mizuno M., Vestibular evoked myogenic potentials in patients with acoustic neuromas. Arch. Otolaryngol. Head Neck Surg. 124: 509512,1998

55. Chen C.W., Young Y.-H. and Tseng H.M., Preoperative versus postoperative role of vestibular-evoked myogenic potentials in cerebellopontine angle tumor. Laryngoscope 112: 267-271, 2002.

56. Patko T., Vidal P.P., Vibert N., Tran Ba Huy P. and de Waele C., Vestibular evoked myogenic potentials in patients suffering from an unilateral acoustic neuroma: a study of 170 patients. Clin. Neurophysiol. 114: 1344-1350, 2003. 